\title{
AN UPPER BOUND FOR THE PERMANENT OF A 3-DIMENSIONAL (0,1)-MATRIX
}

\author{
STEPHEN J. DOW AND PETER M. GIBSON
}

ABSTRACT. Let $A=\left(a_{i j k}\right)$ be a 3-dimensional matrix of order $n$. The permanent of $A$ is defined by

$$
\operatorname{per} A=\sum_{\sigma, \tau \in S_{n}} \prod_{i=1}^{n} a_{i \sigma(i) \tau(i)},
$$

where $S_{n}$ is the symmetric group on $\{1,2, \ldots, n\}$. Suppose that $A$ is a $(0,1)$ matrix and that $r_{i}=\sum_{j, k=1}^{n} a_{i j k}$ for $i=1,2, \ldots, n$. In this paper it is shown that per $A \leq \prod_{i=1}^{n} r_{i} !^{1 / r_{i}}$. A similar bound is then obtained for a second function, the 2-permanent of a 3-dimensional matrix, that is another analogue of the permanent of an ordinary (2-dimensional) matrix.

1. Introduction. It was conjectured by Minc $[\mathbf{3}]$ and proved by Brègman $[\mathbf{1}]$ that if $A$ is a $(0,1)$-matrix of order $n$ with row sums $r_{1}, r_{2}, \ldots, r_{n}$, then

$$
\operatorname{per} A \leq \prod_{i=1}^{n} r_{i} !^{1 / r_{i}}
$$

where by definition $0 !^{1 / 0}=0$. In this paper it is shown that an analogous result holds for 3-dimensional matrices. The permanent of a 3-dimensional matrix $A=$ $\left(a_{i j k}\right)$ of order $n$ is defined by

$$
\operatorname{per} A=\sum_{\sigma, \tau \in S_{n}} \prod_{i=1}^{n} a_{i \sigma(i) \tau(i)}
$$

where $S_{n}$ is the symmetric group on $\{1,2, \ldots, n\}$. We now state our principal result.

THEOREM 1. Let $A=\left(a_{i j k}\right)$ be a 3-dimensional $(0,1)$-matrix of order $n$, and let $r_{i}=\sum_{j, k=1}^{n} a_{i j k}$ for $i=1,2, \ldots, n$. Then

$$
\text { per } A \leq \prod_{i=1}^{n} r_{i} !^{1 / r_{i}}
$$

The proof of Theorem 1, presented in $\S 2$, is an adaptation of Schrijver's proof [4] of the Minc-Brègman bound, with the extension to 3-dimensional matrices requiring a number of additional lemmas which may be of some independent interest.

In equation (1) and Theorem 1 the planes of $A$ play the role of the rows and columns of an ordinary (2-dimensional) matrix, where a plane of a 3-dimensional

Received by the editors December 2, 1985.

1980 Mathematics Subject Classification (1985 Revision). Primary 15A15; Secondary 05A20. 
matrix $A=\left(a_{i j k}\right)$ is a submatrix obtained by fixing one of the indices $i, j, k$ and letting the other two vary. We could also have taken the analogue of rows and columns to be the lines of $A$, obtained by fixing two of these indices. In $\S 3$ we give a second definition of the permanent of a 3-dimensional matrix, called the 2permanent, based on this observation. Using a result from [2] which relates these two types of permanents we obtain an upper bound for the 2-permanent of a 3dimensional $(0,1)$-matrix similar to the one given in Theorem 1 , with plane sums replaced by line sums.

2. Proof of the principal result. We write $N, Z$, and $R^{+}$to denote, respectively, the set of natural numbers, the set of integers, and the set of positive real numbers. Also, for any real number $x$, we use the notation $\lfloor x\rfloor=\max \{k \in Z: k \leq$ $x\}$ and $\lceil x\rceil=\min \{k \in Z: x \leq k\}$. The first lemma appears in [4].

LEMMA 1. If $t=\sum_{i=1}^{n} t_{i}, t_{i} \in R^{+}$, then $t^{t} \leq n^{t} \prod_{i=1}^{n} t_{i}^{t_{i}}$.

LEMMA 2. For all $n \in N, n !^{1 / n}(n+2) !^{1 /(n+2)} \leq(n+1) !^{2 /(n+1)}$.

PROOF. It is well known that if $x_{1}, x_{2}, \ldots, x_{k} \in R^{+}$, then $\prod_{i=1}^{k} x_{i} \leq \bar{x}^{k}$ where $\bar{x}=\left(\sum_{i=1}^{k} x_{i}\right) / k$. Let $x_{i}=i$ for $i=1,2, \ldots, n, x_{i}=i-n$ for $i=n+1, n+2, \ldots, 2 n$, and $x_{i}=n+2$ for $i=2 n+1,2 n+2, \ldots, n^{2}+3 n$. Then

$$
\prod_{i=1}^{n^{2}+3 n} x_{i}=n !^{2}(n+2)^{n^{2}+n}
$$

and

$$
\sum_{i=1}^{n^{2}+3 n} x_{i}=\left(n^{2}+3 n\right)(n+1)
$$

Hence the inequality above becomes $n !^{2}(n+2)^{n^{2}+n} \leq(n+1)^{n^{2}+3 n}$, which is equivalent to the desired inequality.

LEMMA 3. Suppose that a function $f: N \rightarrow R^{+}$has the property that

$$
f(x) f(x+2) \leq(f(x+1))^{2}
$$

for all $x \in N$, and that $x_{1}, x_{2}, \ldots, x_{k} \in N$. Let $\sum_{i=1}^{k} x_{i}=k \bar{x}=k_{1}\lfloor\bar{x}\rfloor+k_{2}\lceil\bar{x}\rceil$, where $k_{1}, k_{2} \in Z, k_{1}, k_{2} \geq 0$, and $k_{1}+k_{2}=k$. Then

$$
\prod_{i=1}^{k} f\left(x_{i}\right) \leq f(\lfloor\bar{x}\rfloor)^{k_{1}} f(\lceil\bar{x}\rceil)^{k_{2}} \text {. }
$$

PROOF. It follows from induction on $n$ that

$$
f(x) f(x+n) \leq f(x+1) f(x+n-1)
$$

for all $x, n \in N$. To see this note that the inductive step is

$$
f(x) f(x+n+1)=\frac{f(x) f(x+2) f(x+1) f(x+1+n)}{f(x+1) f(x+2)} \leq f(x+1) f(x+n) .
$$

By (2), if there exist $p$ and $q$ such that $x_{p}-x_{q}>1$ and we let $x_{p}^{\prime}=x_{p}-1$, $x_{q}^{\prime}=x_{q}+1$, and $x_{i}^{\prime}=x_{i}$ for $i \neq p, q$, then $\prod_{i=1}^{k} f\left(x_{i}\right) \leq \prod_{i=1}^{k} f\left(x_{i}^{\prime}\right)$. By repeating this step the upper bound eventually becomes $f(\lfloor\bar{x}\rfloor)^{k_{1}} f(\lceil\bar{x}\rceil)^{k_{2}}$. 
Lemma 4. Let $f(x)=x !^{1 / x}$ for $x \in N$. Suppose that $n, p, r \in N, n \geq 3$, and $p \leq(r-1) /(n-1)$. Then

$$
f(r-p-1)^{r-p n+p-1} f(r-p)^{(p+1) n-p-r} \leq(r-1) ! r !^{n / r-1} .
$$

ProOF. Let $k \in N, k \leq p$. Then $r-k-1>0$. Therefore, by Lemma 2, $f(r-k-1) f(r-k+1) \leq f(r-k)^{2}$. Since $k \leq(r-1) /(n-1)$, we can raise both sides of this inequality to the power $r-k n+k-1$ to obtain

$$
f(r-k-1)^{r-k n+k-1} f(r-k+1)^{r-k n+k-1} \leq f(r-k)^{2(r-k n+k-1)} .
$$

This inequality is equivalent to

$$
\begin{aligned}
& f(r-k-1)^{r-k n+k-1} f(r-k)^{(k+1) n-k-r} \\
& \quad \leq f(r-k)^{r-(k-1) n+k-2} f(r-k+1)^{k n-k+1-r} .
\end{aligned}
$$

The desired inequality can now be obtained by successively applying (3) with $k=p$, $p-1, p-2, \ldots, 1$. then

LEMMA 5. Let $x_{1}, x_{2}, \ldots, x_{n-1}, r \in N$ with $n>2$. If $\sum_{i=1}^{n-1} x_{i} \leq(n-2) r+1$,

$$
\prod_{i=1}^{n-1} x_{i} !^{1 / x_{i}} \leq(r-1) ! r !^{n / r-1}
$$
Hence

PROOF. Since $x !^{1 / x}$ is increasing in $x$ we may assume that $\sum_{i=1}^{n-1} x_{i}=(n-2) r+1$.

$$
\bar{x}=\frac{(n-2) r+1}{n-1}=r-\frac{r-1}{n-1} .
$$

Let $p=\lfloor(r-1) /(n-1)\rfloor$. Then $\lfloor\bar{x}\rfloor=\lceil\bar{x}\rceil=r-p$ if $x$ is an integer and $\lfloor\bar{x}\rfloor=r-p-1$, $\lceil\bar{x}\rceil=r-p$ otherwise. Since

$$
(r-p n+p-1)(r-p-1)+((p+1) n-p-r)(r-p)=(n-2) r+1
$$

we can apply Lemma 3 with $f(x)=x !^{1 / x}, k=n-1, k_{1}=r-p n+p-1$, and $k_{2}=(p+1) n-p-r$. (When $\lfloor\bar{x}\rfloor=\lceil\bar{x}\rceil=r-p$ we have $k_{1}=0$.) Therefore Lemmas 3 and 4 imply that

$$
\prod_{i=1}^{n-1} x_{i} !^{1 / x_{i}} \leq f(r-p-1)^{r-p n+p-1} f(r-p)^{(p+1) n-p-r} \leq(r-1) ! r !^{n / r-1} .
$$

LEMMA 6. If $B=\left(b_{j k}\right)$ is a $(0,1)$-matrix of order $n$ with $\sum_{j, k=1}^{n} b_{j k}=r$, then

$$
\sum_{i=1}^{n-1} \sum_{\substack{j, k \\ j \neq i \\ k \neq i}} b_{j k} \leq(n-2) r+1 .
$$

ProOF.

$$
\begin{aligned}
\sum_{i=1}^{n-1} \sum_{\substack{j, k \\
j \neq i \\
k \neq i}} b_{j k} & =\sum_{i=1}^{n-1}\left(r-\sum_{j=1}^{n} b_{j i}-\sum_{k=1}^{n} b_{i k}+b_{i i}\right) \\
& =(n-3) r+\sum_{j=1}^{n} b_{j n}+\sum_{k=1}^{n} b_{n k}+\sum_{i=1}^{n-1} b_{i i} \\
& \leq(n-3) r+r+1=(n-2) r+1 .
\end{aligned}
$$


We now show how Schrijver's proof [4] of the Minc-Brègman bound can be altered to prove Theorem 1 .

PROOF OF THEOREM 1. The proof is by induction on $n$. Clearly the theorem holds for $n=1$. Let $n>1$. It suffices to assume that $r_{i} \in N$ for $i=1,2, \ldots, n$. Let $A_{i j k}$ denote the 3-dimensional submatrix of $A$ of order $n-1$ obtained by deleting the three planes through cell $(i, j, k)$. Let

$$
S=\left\{(\sigma, \tau): \prod_{i=1}^{n} a_{i \sigma(i) \tau(i)}=1\right\} .
$$

Then $|S|=\operatorname{per} A$. Apply Lemma 1 with $t=\operatorname{per} A=\sum_{j, k ; a_{i j k}=1} \operatorname{per} A_{i j k}$ for $i=1,2, \ldots, n$, to obtain

$$
\begin{aligned}
(\operatorname{per} A)^{n \text { per } A} & =\prod_{i=1}^{n}(\operatorname{per} A)^{\text {per } A} \\
& \leq \prod_{i=1}^{n} r_{i}^{\text {per } A} \prod_{\substack{j, k \\
a_{i j k}=1}}\left(\operatorname{per} A_{i j k}\right)^{\text {per } A_{i j k}} .
\end{aligned}
$$

For each $i$ we have

$$
\begin{aligned}
\prod_{\substack{j, k \\
a_{i j k}=1}}\left(\operatorname{per} A_{i j k}\right)^{\operatorname{per} A_{i j k}} & =\prod_{\substack{j, k \\
a_{i j k=1}}}\left(\operatorname{per} A_{i j k}\right)^{|\{(\sigma, \tau) \in S: \sigma(i)=j, \tau(i)=k\}|} \\
& =\prod_{(\sigma, \tau) \in S} \operatorname{per} A_{i \sigma(i) \tau(i)} .
\end{aligned}
$$

By the induction hypothesis,

$$
\operatorname{per} A_{i \sigma(i) \tau(i)} \leq \prod_{\substack{p \\ p \neq i}} r(p, i) !^{1 / r(p, i)}
$$

where

$$
r(p, i)=\sum_{\substack{j, k \\ j \neq \sigma(i) \\ k \neq \tau(i)}} a_{p j k}
$$

Combining the inequalities above we have

$$
\begin{aligned}
(\operatorname{per} A)^{n \text { per } A} & \leq \prod_{i=1}^{n} r_{i}^{\text {per } A} \prod_{(\sigma, \tau) \in S} \prod_{\substack{p \\
p \neq i}} r(p, i) !^{1 / r(p, i)} \\
& =\prod_{(\sigma, \tau) \in S}\left(\prod_{i=1}^{n} r_{i}\right) \prod_{i=1}^{n} \prod_{\substack{p \\
p \neq i}} r(p, i) !^{1 / r(p, i)} \\
& =\prod_{(\sigma, \tau) \in S}\left(\prod_{i=1}^{n} r_{i}\right) \prod_{\substack { p=1 \\
\begin{subarray}{c}{i \neq p \\
i \neq p{ p = 1 \\
\begin{subarray} { c } { i \neq p \\
i \neq p } }\end{subarray}} \prod_{\substack{i \\
i}}(p, i) !^{1 / r(p, i)}
\end{aligned}
$$


Fix $\sigma, \tau$, and $p$ and let $b_{j k}=a_{p \sigma(j) \tau(k)}$. Then $\sum_{j, k} b_{j k}=r_{p}$ and for each $i$,

Hence, by Lemma 6,

$$
\sum_{\substack{j, k \\ j \neq i, k \neq i}}=r(p, i) \text {. }
$$

$$
\sum_{\substack{i \\ i \neq p}} r(p, i) \leq(n-2) r_{p}+1
$$

Therefore, by Lemma 5 , if $n>2$, then

$$
\prod_{\substack{i \\ i \neq p}} r(p, i) !^{1 / r(p, i)} \leq\left(r_{p}-1\right) ! r_{p} !^{n / r_{p}-1} .
$$

Moreover, it is easy to see that this inequality also holds for $n=2$. Therefore,

$$
\begin{aligned}
(\operatorname{per} A)^{n \text { per } A} & \leq \prod_{(\sigma, \tau) \in S}\left(\prod_{i=1}^{n} r_{i}\right) \prod_{p=1}^{n}\left(r_{p}-1\right) ! r_{p} !^{n / r_{p}-1} \\
& =\prod_{(\sigma, \tau) \in S} \prod_{p=1}^{n} r_{p} !^{n / r_{p}}=\left(\prod_{i=1}^{n} r_{i} !^{1 / r_{i}}\right)^{n \text { per } A}
\end{aligned}
$$

and the theorem follows.

For some values of the $r_{i}$ it is easy to construct matrices $A$ such that equality holds in Theorem 1. Let $A_{m}=\left(a_{i j k}\right)$ denote the 3-dimensional $(0,1)$-matrix of order $m$ with $a_{i j j}=1$ for $i, j=1,2, \ldots, m$, and $a_{i j k}=0$ otherwise. Then $r_{i}=$ $\sum_{j, k=1}^{n} a_{i j k}=m$ for $i=1,2, \ldots, m$, and per $A_{m}=m$ !. Now let $n_{1}, n_{2}, \ldots, n_{t}$ be a partition of $n$, and let $A$ be the direct sum of $A_{n_{1}}, A_{n_{2}}, \ldots, A_{n_{t}}$. It follows that $A$ is a 3-dimensional $(0,1)$-matrix of order $n$ such that equality holds in Theorem 1 .

3. The 2-permanent. Theorem 1 can be used to obtain another extension of the Minc-Brègman inequality to 3-dimensional matrices. Let $A=\left(a_{i j k}\right)$ be a 3-dimensional matrix of order $n$. The planes of $A$ are the submatrices obtained by fixing one of $i, j, k$, and the lines of $A$ are the submatrices obtained by fixing two of $i, j, k$. Observe that the permanent (or 1-permanent) of $A$ is equal to the summation of all products of $n$ entries of $A$, no two entries from the same plane. Similarly, the 2-permanent of $A$ is defined to be the summation of all products of $n^{2}$ entries of $A$, no two entries from the same line [2]. We have the following upper bound for the 2-permanent of a $(0,1)$-matrix.

THEOREM 2. Let $A=\left(a_{i j k}\right)$ be a 3-dimensional $(0,1)$-matrix of order $n$, and let $r_{i j}=\sum_{k=1}^{n} a_{i j k}$ for $i, j=1,2, \ldots, n$. Then

$$
2 \text {-per } A \leq \prod_{i, j=1}^{n} r_{i j} !^{1 / r_{i j}}
$$

Proof. As shown by the authors [2], there exists a 3-dimensional $(0,1)$-matrix $B=\left(b_{i j k}\right)$ of order $n^{2}$ such that per $B=2$-per $A$. Moreover, $B$ can be chosen so that if $s_{i}=\sum_{j, k=1}^{n^{2}} b_{i j k}$ for $i=1,2, \ldots, n^{2}$, then $\left\{s_{i}: i=1,2, \ldots, n^{2}\right\}=\left\{r_{i j}: i, j=\right.$ $1,2, \ldots, n\}$. Therefore, if Theorem 1 is applied to $B$ the desired upper bound on 2-per $A$ is obtained. 


\section{REFERENCES}

1. L. M. Brègman, Certain properties of nonnegative matrices and their permanents, Dokl. Akad. Nauk SSSR 211 (1973), 27-30; English transl., Soviet Math. Dokl. 14 (1973), 945-949.

2. S. J. Dow and P. M. Gibson, Permanents of d-dimensional matrices (submitted for publication).

3. H. Minc, Upper bounds for permanents of $(0,1)$-matrices, Bull. Amer. Math. Soc. 69 (1963), 789-791.

4. A. Schrijver, A short proof of Minc's conjecture, J. Combin. Theory Ser. A 25 (1978), 80-83.

Department of Mathematics, University of Alabama, Huntsville, Alabama 35899 